\title{
HAT-P-7: A RETROGRADE OR POLAR ORBIT, AND A THIRD BODY
}

\author{
Joshua N. Winn ${ }^{1}$, John Asher Johnson ${ }^{2,7}$, Simon Albrecht ${ }^{1}$, Andrew W. Howard ${ }^{3,4,8}$, Geoffrey W. Marcy ${ }^{3}$, \\ Ian J. CRossfield ${ }^{5}$, and Matthew J. Holman ${ }^{6}$ \\ ${ }^{1}$ Department of Physics, and Kavli Institute for Astrophysics and Space Research, Massachusetts Institute of Technology, Cambridge, MA 02139, USA \\ ${ }^{2}$ Institute for Astronomy, University of Hawaii, Honolulu, HI 96822, USA \\ ${ }^{3}$ Department of Astronomy, University of California, Mail Code 3411, Berkeley, CA 94720, USA \\ ${ }^{4}$ Space Sciences Laboratory, University of California, Berkeley, CA 94720, USA \\ ${ }^{5}$ Department of Physics and Astronomy, University of California, Los Angeles, CA 90095, USA \\ ${ }^{6}$ Harvard-Smithsonian Center for Astrophysics, 60 Garden St., Cambridge, MA 02138, USA \\ Received 2009 August 12; accepted 2009 August 21; published 2009 September 8
}

\begin{abstract}
We show that the exoplanet HAT-P-7b has an extremely tilted orbit, with a true angle of at least $86^{\circ}$ with respect to its parent star's equatorial plane, and a strong possibility of retrograde motion. We also report evidence for an additional planet or companion star. The evidence for the unparalleled orbit and the third body is based on precise observations of the star's apparent radial velocity (RV). The anomalous RV due to rotation (the Rossiter-McLaughlin effect) was found to be a blueshift during the first half of the transit and a redshift during the second half, an inversion of the usual pattern, implying that the angle between the sky-projected orbital and stellar angular momentum vectors is $182.5 \pm 9.4$. The third body is implicated by excess RV variation of the host star over $2 \mathrm{yr}$. Some possible explanations for the tilted orbit of HAT-P-7b are a close encounter with another planet, the Kozai effect, and resonant capture by an inward-migrating outer planet.
\end{abstract}

Key words: planetary systems - planetary systems: formation - stars: individual (HAT-P-7) - stars: rotation

Online-only material: machine-readable table

\section{INTRODUCTION}

In the solar system, the planetary orbits are well aligned and prograde, revolving in the same direction as the rotation of the Sun. This fact inspired the "nebular hypothesis" that the Sun and planets formed from a single spinning disk (Laplace 1796). One might also expect exoplanetary orbits to be well aligned with their parent stars, and indeed this is true of most systems for which it has been possible to compare the directions of orbital motion and stellar rotation (Fabrycky \& Winn 2009; Le Bouquin et al. 2009). However, there are at least three exoplanets for which the orbit is tilted by a larger angle than any of the planets in the solar system: XO-3b (Hébrard et al. 2008; Winn et al. 2009a), HD 80606b (Moutou et al. 2009; Pont et al. 2009; Winn et al. 2009b), and WASP-14b (Johnson et al. 2009).

Still, all of those systems are consistent with prograde orbits, with the largest minimum angle between the stellar-rotational and orbital angular momentum vectors of about $37^{\circ}$, for XO-3b (Winn et al. 2009a). The reason why only the minimum angle is known is that the evidence for misalignment is based on the eponymous effect of Rossiter (1924) and McLaughlin (1924), an anomalous Doppler shift observed during planetary transits that is sensitive only to the angle between the sky projections of the two vectors. The true spin-orbit angle may be larger, depending on the unknown inclination angle of the stellar rotation axis with respect to the line of sight.

In this Letter, we present evidence of a very large spin-orbit misalignment for HAT-P-7b, a planet of mass $1.8 M_{\text {Jup }}$ and radius $1.4 R_{\mathrm{Jup}}$ in a 2.2-day orbit around an F6V star with mass $1.5 M_{\odot}$ and radius $1.8 R_{\odot}$ (Pál et al. 2008). We find the angle between the sky-projected angular momentum vectors to be $182.5 \pm 9.4$. Furthermore, we show that the true angle $\psi$ between those

\footnotetext{
7 NSF Astronomy and Astrophysics Postdoctoral Fellow.

8 Townes Postdoctoral Fellow.
}

vectors is likely greater than $86^{\circ}$, indicating that the orbit is either retrograde $\left(\psi>90^{\circ}\right)$ or nearly polar $\left(\psi \approx 90^{\circ}\right)$. We also present evidence for a third body in the system, which may be an additional planet or a companion star. We present spectroscopic data in Section 2, photometric data in Section 3, a joint analysis of both types of data in Section 4, and a discussion of the results in Section 5.

\section{RADIAL VELOCITIES}

We observed HAT-P-7 with the High Resolution Spectrograph (HIRES) on the Keck I 10 m telescope, and the High Dispersion Spectrograph (HDS) on the Subaru $8 \mathrm{~m}$ telescope. The planet's discoverers (Pál et al. 2008; hereafter P08) obtained eight HIRES spectra in 2007, to which we add nine spectra from 2009. All but one of the HIRES spectra were acquired outside of transits. Of the 49 HDS spectra, 9 were obtained on 2009 June 17 and 40 were obtained on 2009 July 1. The second of these nights spanned a transit.

The instrument settings and observing procedures in both 2007 and 2009 were identical to those used by the California Planet Search (CPS; Howard et al. 2009). We placed an iodine gas absorption cell into the optical path, to calibrate the instrumental response and wavelength scale. The radial velocity (RV) of each spectrum was measured with respect to an iodinefree template spectrum, using the algorithm of Butler et al. (2006) with subsequent improvements. Measurement errors were estimated from the scatter in the fits to individual spectral segments spanning a few angstroms. The RVs are given in Table 1.

\subsection{Evidence for a Third Body}

Figure 1 shows the RVs over the two-year span of the observations. Figure 2 shows the RVs as a function of orbital phase, fitted with two different models. The first model is a single 


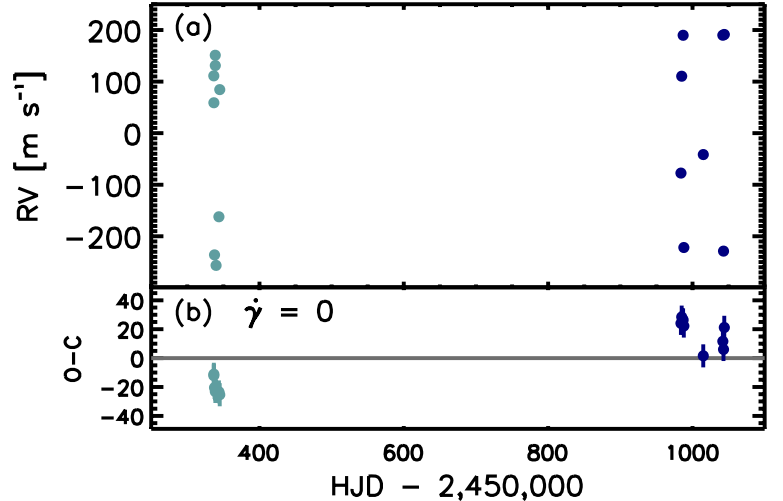

Figure 1. Long-term radial velocity variation of HAT-P-7. (a) Measured RVs. (b) Residuals (observed - calculated) between the data and the best-fitting single-planet model. Light blue and dark blue points are HIRES data from 2007 and 2009 , respectively.

Table 1

Relative Radial Velocity Measurements of HAT-P-7

\begin{tabular}{crcc}
\hline \hline HJD & RV $\left(\mathrm{m} \mathrm{s}^{-1}\right)$ & Error $\left(\mathrm{m} \mathrm{s}^{-1}\right)$ & Spectrograph $^{\mathrm{a}}$ \\
\hline 2454336.73960 & 111.08 & 1.72 & 1 \\
2454336.85367 & 58.89 & 1.78 & 1 \\
2454337.76212 & -236.06 & 1.70 & 1 \\
2454338.77440 & 151.06 & 1.54 & 1 \\
2454338.85456 & 131.12 & 1.57 & 1 \\
\hline
\end{tabular}

Notes. The RV was measured relative to an arbitrary template spectrum specific to each spectrograph; only the differences among the RVs from a single spectrograph are significant. The uncertainty given in Column 3 is the internal error only and does not account for any possible "stellar jitter."

a (1) Keck/HIRES, (2) Subaru/HDS.

(This table is available in its entirety in a machine-readable form in the online journal. A portion is shown here for guidance regarding its form and content.)

Keplerian orbit, representing the signal of the known planet. The second model has an additional parameter $\dot{\gamma}$ representing an extra radial acceleration. The second model gives a better fit to the data, with a root-mean-squared (rms) residual of $7 \mathrm{~m}$ $\mathrm{s}^{-1}$ as compared to $21 \mathrm{~m} \mathrm{~s}^{-1}$ for the first model. The RVs from 2009 are systematically redshifted by approximately $40 \mathrm{~m} \mathrm{~s}^{-1}$ compared to RVs from 2007, as evident from the residuals shown in Figures 1(b) and 2(b). This shift is highly significant, as the CPS has demonstrated a long-term stability of $2 \mathrm{~m} \mathrm{~s}^{-1}$ or better using HIRES and the same reduction codes used here (Howard et al. 2009).

This RV trend is evidence for an additional companion. Given the limited time coverage of our observations (two clusters of points separated by $2 \mathrm{yr}$ ), the data are compatible with nearly any period longer than a few months. A constant acceleration is the simplest model that fits the excess RV variability, and under that assumption we may give an order-of-magnitude relation relating $\dot{\gamma}$ to some properties of the companion

$$
\frac{M_{c} \sin i_{c}}{a_{c}^{2}} \sim \frac{\dot{\gamma}}{G}=(0.121 \pm 0.014) M_{\mathrm{Jup}} \mathrm{AU}^{-2},
$$

where $M_{c}$ is the companion mass, $i_{c}$ its orbital inclination relative to the line of sight, $a_{c}$ its orbital distance, and the numerical value is based on our model-fitting results (see Section 4).

\subsection{Evidence for a Spin-orbit Misalignment}

Figure 3(a) shows the RV data spanning the transit, after subtracting the orbital RV as computed with the best-fitting
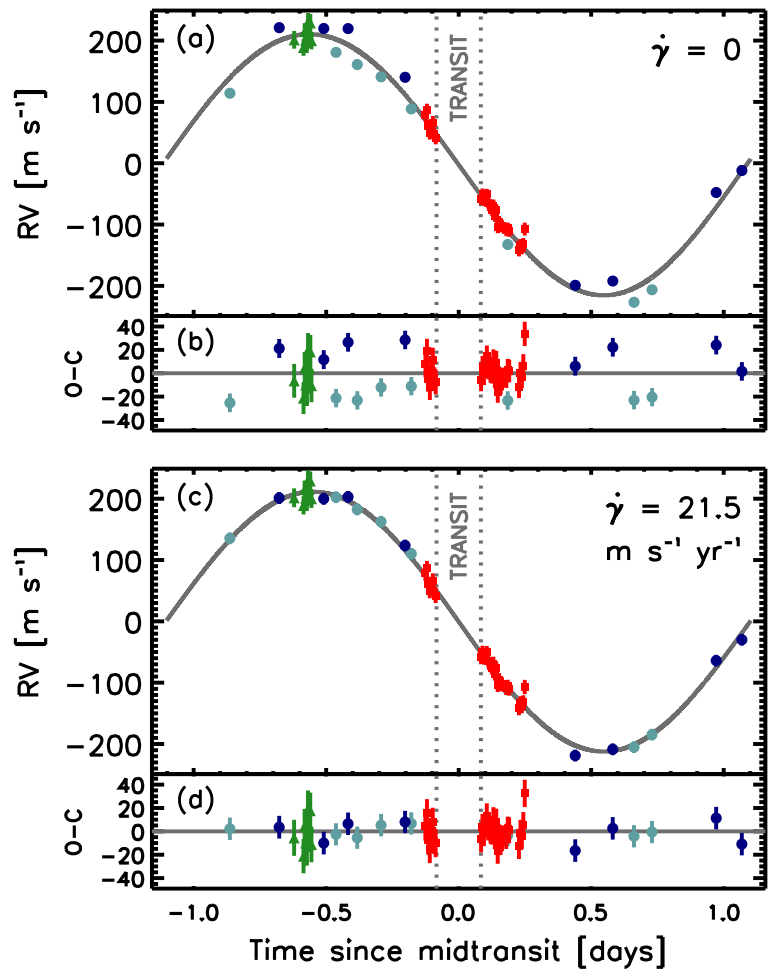

Figure 2. Phased radial velocity variation of HAT-P-7. (a) Assuming a single Keplerian orbit. (b) Residuals. (c) With an extra parameter $\dot{\gamma}$ representing a constant radial acceleration. (d) Residuals. The circles are HIRES data (light blue from 2007, dark blue from 2009), the green triangles are HDS data from 2009 June 17, and the red squares are HDS data from 2009 July 1.

model including $\dot{\gamma}$. We interpret the "anomalous" RV variation during the transit as the Rossiter-McLaughlin (RM) effect, the asymmetry in the spectral lines due to the partial eclipse of the rotating photosphere. In the context of eclipsing binary stars, the RM effect was predicted by Holt (1893) and observed definitively by Rossiter (1924) and McLaughlin (1924). For exoplanets, the RM effect was first observed by Queloz et al. (2000), and its use in assessing spin-orbit alignment has been expounded by Ohta et al. (2005) and Gaudi \& Winn (2007).

A transiting planet in a well-aligned prograde orbit would first pass in front of the blueshifted (approaching) half of the star, causing an anomalous redshift of the observed starlight. Then, the planet would cross to the redshifted (receding) half of the star, causing an anomalous blueshift. In contrast, Figure 3(a) shows a blueshift followed by a redshift: an inversion of the effect just described. We may conclude, even without any modeling, that the orbital "north pole" and the stellar "north pole" point in nearly opposite directions on the sky.

\section{PHOTOMETRY}

For a quantitative analysis of the RM effect, we wanted to model both the photometric and spectroscopic transit signals. For this purpose, we supplemented the RV data with the most precise transit light curve available to us, shown in Figure 3(c). This light curve is based on observations on UT 2008 September 22 in the Sloan $i$ bandpass, with the Fred L. Whipple $1.2 \mathrm{~m}$ telescope and Keplercam detector, under the auspices of the Transit Light Curve project (Holman et al. 2006; Winn et al. 2007).

Reduction of the CCD images involved standard procedures for bias subtraction and flat-field division. Differential aperture 

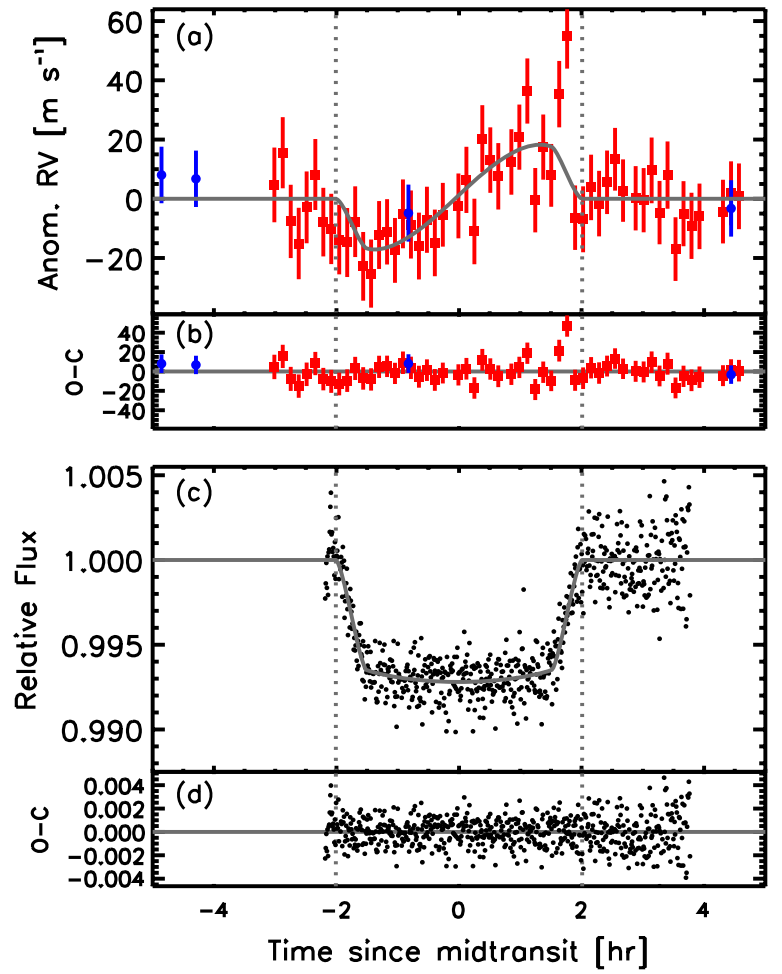

Figure 3. Spectroscopic and photometric transit of HAT-P-7b. (a) The anomalous RV, defined as the output of the Doppler code minus the orbital RV. We observed a blueshift in the first half of the transit, and a redshift in the second half of the transit, demonstrating that the sky projections of the orbital and stellar angular momentum vectors point in opposite directions. (b) Residuals. Red squares are HDS data from 2009 July 1, and blue circles are HIRES data obtained on various nights in 2007 and 2009. (c) The relative flux, observed in the Sloan $i$ band with the FLWO 1.2 m telescope and Keplercam. (d) Residuals. In panels (a) and (b), the gray line shows the best-fitting model.

photometry was performed for HAT-P-7 and seven comparison stars. No evidence was found for time-correlated noise using the "time-averaging" method of Pont et al. (2006), as implemented by Winn et al. (2009c). The data shown in Figure 3(c) were corrected for differential extinction as explained in Section 4.

\section{JOINT ANALYSIS}

We fitted a model to the photometric and RV data in order to derive quantitative constraints on the angle $\lambda$ between the sky projections of the orbital and stellar-rotational angular momentum vectors. This angle is defined such that $\lambda=0^{\circ}$ when the sky-projected vectors are parallel and $\lambda=180^{\circ}$ when they are antiparallel. Our model for the RM effect was based on the technique of Winn et al. (2005): we simulated spectra exhibiting the RM effect at various transit phases, and then measured the apparent RV of the simulated spectra using the same Doppler code that is used on actual data. This allowed us to relate the anomalous RV to the parameters and positions of the star and planet.

The RV model was the sum of the Keplerian RV and the anomalous RV due to the RM effect. The photometric model was based on the analytic equation for the flux of a quadratically limb-darkened disk with a circular obstruction (Mandel \& Agol 2002). As a compromise between fixing the limb-darkening coefficients $u_{1}$ and $u_{2}$ at theoretically calculated values, and giving them complete freedom, we fixed $u_{1}-u_{2}$ at the tabulated value of 0.3846 (Claret 2004) and allowed $u_{1}+u_{2}$ to be a free
Table 2

Model Parameters for HAT-P-7b

\begin{tabular}{lc}
\hline \hline \multicolumn{1}{c}{ Parameter } & Value \\
\hline Orbital period, $P(\mathrm{~d})$ & $2.2047304 \pm 0.0000024$ \\
Midtransit time (HJD) & $2454,731.67929 \pm 0.00043$ \\
Transit duration (first to fourth contact) $(\mathrm{hr})$ & $4.006 \pm 0.064$ \\
Transit ingress or egress duration $(\mathrm{hr})$ & $0.474_{-0.093}^{+0.061}$ \\
\hline Planet-to-star radius ratio, $R_{p} / R_{\star}$ & $0.0834_{-0.0021}^{+0.0012}$ \\
Orbital inclination, $i$ (deg) & $80.8_{-1.2}^{+2.8}$ \\
Scaled semimajor axis, $a / R_{\star}$ & $3.82_{-0.16}^{+0.39}$ \\
Transit impact parameter & $0.618_{-0.149}^{+0.039}$ \\
\hline Velocity semiamplitude, $K\left(\mathrm{~m} \mathrm{~s}^{-1}\right)$ & $211.8 \pm 2.6$ \\
Upper limit on eccentricity $\left(99.73 \% \mathrm{conf}^{-}\right)$ & 0.039 \\
$e$ cos $\omega$ & $-0.0019 \pm 0.0077$ \\
$e$ sin $\omega$ & $0.0037 \pm 0.0124$ \\
Velocity offset, Keck/HIRES $\left(\mathrm{m} \mathrm{s}^{-1}\right)$ & $-51.2 \pm 3.6$ \\
Velocity offset, Subaru/HDS $\left(\mathrm{m} \mathrm{s}^{-1}\right)$ & $-4.8 \pm 2.5$ \\
Constant radial acceleration $\dot{\gamma}\left(\mathrm{m} \mathrm{s}^{-1} \mathrm{yr}^{-1}\right)$ & $21.5 \pm 2.6$ \\
\hline Projected stellar rotation rate, $v \sin _{\star}\left(\mathrm{km} \mathrm{s}^{-1}\right)$ & $4.9_{-0.9}^{+1.2}$ \\
Projected spin-orbit angle, $\lambda\left(\mathrm{deg}^{-1}\right.$ & $182.5 \pm 9.4$ \\
\hline
\end{tabular}

parameter. We also included a free parameter for the coefficient of differential air-mass extinction between HAT-P-7 and the ensemble of comparison stars.

We determined the best values of the model parameters and their $68.3 \%$ confidence limits using a Markov Chain Monte Carlo algorithm, as described in our previous works (see, e.g., Winn et al. 2009a). The likelihood function was given by $\exp \left(-\chi^{2} / 2\right)$ with

$\chi^{2}=\sum_{i=1}^{N_{f}}\left[\frac{f_{i}(\mathrm{obs})-f_{i}(\mathrm{calc})}{\sigma_{f, i}}\right]^{2}+\sum_{i=1}^{N_{v}}\left[\frac{v_{i}(\mathrm{obs})-v_{i}(\mathrm{calc})}{\sigma_{v, i}}\right]^{2}$,

in a self-explanatory notation, with $\sigma_{f, i}$ chosen to be 0.00136 , and $\sigma_{v, i}$ chosen to be the quadrature sum of the RV measurement error and a "stellar jitter" term of $9.3 \mathrm{~m} \mathrm{~s}^{-1}$. These choices led to $\chi^{2}=N_{\text {dof }}$ for the minimum- $\chi^{2}$ model. A Gaussian prior constraint was imposed upon the orbital period based on the precise measurement of P08.

Table 2 gives the results for the model parameters. In particular, the result for $\lambda$ is $182.5 \pm 9.4$, close to antiparallel, as anticipated from the qualitative discussion of Section 2.

\section{DISCUSSION}

Our finding for $\lambda$ is strongly suggestive of retrograde motion, in which the orbital motion and stellar rotation are in opposite directions. However, it must be remembered that $\lambda$ refers to the angle between the sky-projected angular momentum vectors. The true angle $\psi$ between the vectors is given by

$$
\cos \psi=\cos i_{\star} \cos i+\sin i_{\star} \sin i \cos \lambda,
$$

where $i$ and $i_{\star}$ are the line-of-sight inclinations of the orbital and stellar angular momentum vectors, respectively. Although $i$ is known precisely from the transit data, $i_{\star}$ is unknown.

Supposing $i_{\star}$ to be drawn from an "isotropic" distribution (uniform in $\cos i_{\star}$ ), the data demand that $\psi>86.3$ with $99.73 \%$ confidence. Thus, under this assumption, a retrograde orbit is strongly favored, although a nearly polar and barely prograde orbit cannot be ruled out. 
In fact, there is circumstantial evidence that $i_{\star}$ is small and consequently the orbit of HAT-P-7b is nearly polar $\left(\psi \approx 90^{\circ}\right)$. The star's projected rotation rate is unusually low for such a hot star: $v \sin i_{\star}=4.9_{-0.9}^{+1.2} \mathrm{~km} \mathrm{~s}^{-1}$ in our model, or $3.8 \pm 0.5 \mathrm{~km} \mathrm{~s}^{-1}$ based on the line profile analysis of P08, and $T_{\text {eff }}=6350 \pm 80 \mathrm{~K}$ according to P08. In the SPOCS catalog of dwarf stars with welldetermined spectroscopic properties (Valenti \& Fischer 2005), only 2 of 37 stars with $T_{\text {eff }}=6350 \pm 100 \mathrm{~K}$ have $v \sin i_{\star}<$ $4.9 \mathrm{~km} \mathrm{~s}^{-1}$.

Based on this catalog, the mean rotation rate $v$ for such hot stars is about $15 \mathrm{~km} \mathrm{~s}^{-1}$. As an alternate approach to constraining $\psi$, we assumed the rotation velocity $v$ is drawn from a Gaussian distribution with mean $15 \mathrm{~km} \mathrm{~s}^{-1}$ and standard deviation $3 \mathrm{~km} \mathrm{~s}^{-1}$. The result is $\psi=94.6_{-3.0}^{+5.5}$ deg with $68.3 \%$ confidence, and $\psi>86.1$ with $99.73 \%$ confidence. This analysis favors nearly polar and retrograde orbits. However, one wonders whether HAT-P-7 should be expected to have a "typical" rotation rate, given the existence of its short-period planet on a bizarre orbit. Another caveat is that we found the scaled semimajor axis $a / R_{\star}$ to be about $1 \sigma$ smaller than the finding of P08, suggesting the star is somewhat larger and more evolved, which would correspond to a slower expected rotation rate.

Determining $i_{\star}$ directly may be possible by measuring and interpreting asteroseismological oscillations (Gizon \& Solanki 2003), or photometric modulations produced by starspots (see, e.g., Henry \& Winn 2008). By good fortune, HAT-P-7 is in the field of view of the Kepler satellite, which is capable of precise long-term photometry and may be able to accomplish these tasks (Borucki et al. 2009).

The extraordinary orbit of HAT-P-7b presents an extreme case for theories of planet formation and subsequent orbital evolution. HAT-P-7b is a "hot Jupiter" and presumably migrated inward toward the star after its formation. A prevailing migration theory involves tidal interactions with the protoplanetary disk, but such interactions would probably not perturb the initial coplanarity of the system, and might even bring the system into closer alignment (Lubow \& Ogilvie 2001; Cresswell et al. 2007). More promising to explain HAT-P-7b are scenarios involving few-body dynamics, as those scenarios are expected to produce misalignments. In one scenario, close encounters between planets throw a planet inward, where its orbit is ultimately shrunk and circularized by tidal dissipation (Chatterjee et al. 2008; Jurić \& Tremaine 2008). Another idea is based on the Kozai (1962) effect, whereby the gravitational force from a distant body on a highly inclined orbit strongly modulates an inner planet's orbital eccentricity and inclination (Fabrycky \& Tremaine 2007). Recent calculations showed that a combination of planet-planet scattering, the Kozai effect, and tidal friction can lead to nearly circular retrograde orbits (Nagasawa et al. 2008). A third proposed scenario involves an inward-migrating outer planet that captures an inner planet into a mean motion resonance; if the inner planet avoids being ejected or consumed by the star, it may be released on a nearly circular retrograde orbit (Yu \& Tremaine 2001).

The prospect of explaining HAT-P-7b's orbit through fewbody dynamics lends extra importance to measuring the mass and orbital parameters of the third body. If it turns out to be a planet, then HAT-P-7b will be only the second known case of a transiting planet accompanied by another planet, the first being HAT-P-13b (Bakos et al. 2009). Such systems are highly desirable because the unusually precise measurements enabled by transit observations can be used to determine whether the orbits are coplanar and give clues about the system's dynamical history (Fabrycky 2009).
We are grateful to Yasushi Suto and Ed Turner for stimulating our interest in this subject; Norio Narita and his team for sharing their data in advance of publication; Dan Fabrycky, András Pál, Darin Raggozine, Scott Tremaine, Bill Welsh, and the anonymous referee for helpful comments on the manuscript; Akito Tajitsu, Tae-Soo Pyo, Mark Everett, Howard Isaacson, and Zach Gazak for assistance with observing; Gáspár Bakos and Joel Hartman for help obtaining telescope time; Eric Gaidos and Debra Fischer for trading telescope time on short notice; and Hector Balbontin for hospitality at Las Campanas Observatory where this manuscript was written.

Some of the data presented herein were obtained at the W. M. Keck Observatory, which is operated as a scientific partnership among the California Institute of Technology, the University of California, and the National Aeronautics and Space Administration, and was made possible by the generous financial support of the W. M. Keck Foundation. We extend special thanks to those of Hawaiian ancestry on whose sacred mountain of Mauna Kea we are privileged to be guests. Without their generous hospitality, the Keck observations presented herein would not have been possible. J.A.J. gratefully acknowledges support from the NSF Astronomy and Astrophysics Postdoctoral Fellowship program (grant AST-0702821). S.A. acknowledges the support of the Netherlands Organisation for Scientific Research (NWO). J.N.W. gratefully acknowledges support from the NASA Origins program through awards NNX09AD36G and NNX09AB33G, and from an MIT Class of 1942 Career Development Professorship.

Facilities: Subaru (HDS), Keck I (HIRES), FLWO $1.2 \mathrm{~m}$ (Keplercam)

Note added after submission. Narita et al. (2009) report independent evidence for a retrograde or polar orbit of HAT-P$7 \mathrm{~b}$, based on Subaru/HDS spectra spanning the transit of 2008 May 30.

\section{REFERENCES}

Bakos, G. A., et al. 2009, arXiv:0907.3525

Borucki, W. J., et al. 2009, Science, 325, 709

Butler, R. P., et al. 2006, ApJ, 646, 505

Chatterjee, S., Ford, E. B., Matsumura, S., \& Rasio, F. A. 2008, ApJ, 686, 580

Claret, A. 2004, A\&A, 428, 1001

Cresswell, P., Dirksen, G., Kley, W., \& Nelson, R. P. 2007, A\&A, 473, 329

Fabrycky, D. C. 2009, in IAU Symp. 253, ed. F. Pont, D. D. Sasselov, \& M. J. Holman (Cambridge: Cambridge Univ. Press), 173

Fabrycky, D., \& Tremaine, S. 2007, ApJ, 669, 1298

Fabrycky, D. C., \& Winn, J. N. 2009, ApJ, 696, 1230

Gaudi, B. S., \& Winn, J. N. 2007, ApJ, 655, 550

Gizon, L., \& Solanki, S. K. 2003, ApJ, 589, 1009

Hébrard, G., et al. 2008, A\&A, 488, 763

Henry, G. W., \& Winn, J. N. 2008, AJ, 135, 68

Holman, M. J., et al. 2006, ApJ, 652, 1715

Holt, J. R. 1893, A\&A, 12, 646

Howard, A. W., et al. 2009, ApJ, 696, 75

Johnson, J. A., Winn, J. N., Albrecht, S., Howard, A. W., Marcy, G. W., \& Gazak, J. Z. 2009, PASP, in press (arXiv:0907.5204)

Jurić, M., \& Tremaine, S. 2008, ApJ, 686, 603

Kozai, Y. 1962, AJ, 67, 591

Laplace, P. S. 1796, Exposition du Système du Monde (Paris: Cercle-Social) Le Bouquin, J.-B., Absil, O., Benisty, M., Massi, F., Mérand, A., \& Stefl, S. 2009, A\&A, 498, L41

Lubow, S. H., \& Ogilvie, G. I. 2001, ApJ, 560, 997

Mandel, K., \& Agol, E. 2002, ApJ, 580, L171

McLaughlin, D. B. 1924, ApJ, 60, 22

Moutou, C., et al. 2009, A\&A, 498, L5

Nagasawa, M., Ida, S., \& Bessho, T. 2008, ApJ, 678, 498 
Narita, N., Sato, B., Hirano, T., \& Tamura, M. 2009, PASJ, in press (arXiv:0908.1673)

Ohta, Y., Taruya, A., \& Suto, Y. 2005, ApJ, 622, 1118

Pál, A., et al. 2008, ApJ, 680, 1450

Pont, F., Zucker, S., \& Queloz, D. 2006, MNRAS, 373, 231

Pont, F., et al. 2009, A\&A, 502, 695

Queloz, D., Eggenberger, A., Mayor, M., Perrier, C., Beuzit, J. L., Naef, D., Sivan, J. P., \& Udry, S. 2000, A\&A, 359, L13
Rossiter, R. A. 1924, ApJ, 60, 15

Valenti, J. A., \& Fischer, D. A. 2005, ApJS, 159, 141

Winn, J. N., Holman, M. J., \& Fuentes, C. I. 2007, AJ, 133, 11

Winn, J. N., et al. 2005, ApJ, 631, 1215

Winn, J. N., et al. 2009a, ApJ, 700, 302

Winn, J. N., et al. 2009b, ApJ, in press (arXiv:0907.5205)

Winn, J. N., et al. 2009c, ApJ, 693, 794

Yu, Q., \& Tremaine, S. 2001, AJ, 121, 1736 